\title{
The Ethics of Mandatory Retention of Clinical Biospecimens for Research
}

\author{
David Wendler, PhD® \\ Section on Research Ethics, Department of Bioethics, National Institutes of Health, \\ Bethesda, MD, USA.
}

J Gen Intern Med 36(9):2818-9

DOI: $10.1007 / \mathrm{s} 11606-020-06468-9$

(c) Society of General Internal Medicine 2021

B illions of biospecimens are stored in the USA and around the world. Together with associated clinical data, these specimens offer an "invaluable resource for current and future research."1 To realize this potential, many hospitals and clinics retain biospecimens left over from clinical care and make them available for research. ${ }^{1}$ Increasingly, this practice is being implemented by means of a mandate which gives prospective patients the choice of allowing their left-over samples to be retained, or seeking care elsewhere. The present manuscript evaluates whether this practice represents an appropriate way to maximize the social value of biorepositories or a failure to respect patients appropriately.

\section{WHEN SHOULD CONSENT BE OBTAINED?}

Under US regulations (45CFR46), storage of identifiable samples for future research itself constitutes research, which requires extensive consent (or waiver of consent). When samples are not identifiable, their retention does not constitute research. For example, if samples are anonymized, or coded in a way that investigators cannot readily ascertain the donors' identities, their retention is not subject to US regulations. Hence, unless state law specifies otherwise, institutions can decide whether to obtain consent to retain samples for future research.

Because the use of left-over samples does not require individuals to do anything extra, one might conclude that there is no need to obtain any consent to retain left-over samples which are not identifiable. Yet, obtaining consent allows individuals to decide whether they contribute to future research through their samples. In addition, obtaining consent shows

Prior Presentations A previous version of this work was presented as Grand Rounds at Northwestern Medical Center/Lurie Children's Hospital, March 4, 2020

Received August 25, 2020

Accepted December 13, 2020

Published online January 26, 2021 respect for the many individuals who want to decide whether their samples are used for research. Finally, obtaining consent provides a way to maintain trust in the institution, and in research more generally.

These considerations suggest that, when feasible, institutions should obtain consent to retain samples for future research, even when the samples are not identifiable. ${ }^{2}$ This raises the question of which approach to consent is preferable.

\section{THE ETHICS OF MANDATORY RETENTION}

Increasingly, institutions obtain consent by means of a mandate which informs individuals that signing the admission form constitutes their agreement to the institution retaining any left-over samples for future research. This practice enhances the value of biorepositories, eliminates the need to track the samples of individuals who decline research use, and allows those who have strong objections to seek care elsewhere. Moreover, appropriate practice places many conditions on patient care which are designed to benefit others. ${ }^{3}$ To consider just a few, patients must come during clinic hours, accept only drugs on the formulary, and agree to be cared for based on institution protocols. Many institutions also inform individuals that signing the admission form constitutes their agreement to care being provided by inexperienced trainees.

These examples reveal that placing conditions on patients' access to care does not necessarily conflict with respect for patient autonomy. At the same time, conditions on access to care should satisfy at least three conditions. First, the mandated practice should be appropriate. For example, surgeons may mandate that patients accept a certain type of scalpel; surgeons may not mandate that patients undergo surgery naked. The widespread acceptance of research using biospecimens suggests it satisfies this condition.

Second, the mandated practice should pose low risks. Some commentators argue that future research on biospecimens can pose significant risks. ${ }^{4}$ In fact, as long as standard safeguards are employed, research using left-over samples poses less than a 1 in a million chance of serious harm. ${ }^{5}$ These data reveal that, even granting increased capacity to re-identify samples, research on biospecimens poses extremely low risks.

Third, conditions should be placed on access to care only when needed to realize a valuable goal. In this regard, 
individuals are frequently willing to have their samples used for research. This raises the possibility that a mandate may not be needed to obtain a sufficient number of samples. For example, one study found that use of opt-out consent resulted in $95 \%$ of individuals permitting their samples to be used for research. $^{6}$

Before adopting mandatory retention, institutions should assess whether opt-in or opt-out consent would yield sufficient samples. They should also consider the feasibility of tracking the samples of individuals who decline. When these considerations suggest that opt-in or opt-out consent offers a feasible means to collecting sufficient samples, a mandate should not be used.

When feasibly obtaining sufficient samples requires a mandate, the potential for samples to be used in research which helps to improve health and well-being provides an important ethical argument in favor of the mandate. This, combined with the fact that use of samples poses extremely low risks and does not require individuals to do anything extra, suggests that, on balance, the ethical considerations support the use of a mandate in these cases. When they do, how should a mandate be implemented?

\section{IMPLEMENTING A MANDATE}

Some individuals have fundamental objections (e.g., religious) to their left-over samples being used for research. For these individuals, a mandate represents a significant obstacle to obtaining clinical care. This is an important concern when individuals have limited options due to geographic limitations, insurance requirements, rarity of illness, or other factors. Recognizing this possibility, a mandate should include a process which allows individuals, including those who are too ill to make this decision at admission, to opt out. This process should be designed so that it does not pose significant burdens on patients. Assuming only a few individuals have fundamental objections, this option should have minimal impact on the value of the repository.

Given that mandated retention affects the entire community, it should be transparent to the public. ${ }^{7}$ Transparency ensures that individuals are aware of the practice and allows those with fundamental objections to seek care elsewhere or opt out. In addition, institutions should engage the community as partners in the research enterprise. An advisory board could provide insight and recommendations on the types of research that would be valuable, and any that might be objectionable. A dedicated website which informs the community of the studies it has supported would provide additional means for input on any the community finds objectionable.

Finally, samples obtained through a mandate should be used only for research that has important social value, is ethically appropriate, and does not harm individuals or the groups to which they belong. To ensure these conditions are satisfied, institutions should mandate that all research uses of collected samples undergo independent review and approval.

Acknowledgments: Thanks are due to Robert Steel, Christine Grady, Seema Shah, and the participants at Northwestern Medical Center/ Lurie Children's Hospital for their valuable suggestions.

Corresponding Author: David Wendler, PhD; Section on Research Ethics, Department of Bioethics, National Institutes of Health, Bethesda, MD, USA (e-mail: dwendler@nih.gov).

Funding The present work was funded by intramural funds of the NIH Clinical Center.

\section{Compliance with Ethical Standards:}

Conflict of Interest: The author declares that he does not have a conflict of interest.

Disclaimer: The views expressed are the author's own. They do not represent the position or the policy of the NIH or the Department of Health and Human Services.

\section{REFERENCES}

1. SACHRP. Attachment C - Updated FAQs on Informed Consent for Use of Biospecimens and Data. Available at: https://www.hhs.gov/ohrp/sachrpcommittee/recommendations/attachment-c-faqs-recommendations-andglossary-informed-consent-and-research-use-of-biospecimens-and-associated-data/index.html.

2. Wolinetz CD, Collins FS. Recognition of research participants' need for autonomy remembering the legacy of Henrietta Lacks [Published online August 11, 2020]. JAMA. https://doi.org/10.1001/jama.2020.15936.

3. Wendler $\mathbf{D}$. Are physicians obligated always to act in the patient's best interests? J Med Ethic. 2010;36:66-70.

4. Edwards KL, et al. Genetics researchers' and IRB professionals' attitudes toward genetic research review: a comparative analysis. Genet Med. 2012;14:236-42.

5. Wendler D, Rid A. Genetic research on biospecimens poses minimal risk. Trends Genet. 2015;31:11-5.

6. Brothers KB, Morrison DR, Clayton EW. Two large-scale surveys on community attitudes toward an opt-out biobank. Am J Med Genet. 2011;155A:2982-90.

7. Botkin JR, Rothwell E, Anderson R, Stark LA, Mitchell J. Public attitudes regarding the use of electronic health information and residual clinical tissues for research. J Commun Genet. 2014;5:205-13.

Publisher's Note: Springer Nature remains neutral with regard to jurisdictional claims in published maps and institutional affiliations. 